\title{
Educación financiera de jóvenes universitarios de la Facultad de Humanidades y Ciencias de la Educación, periodo 2019-2020
}

\author{
Amelio Florentín González Vázquez \\ ameliogonzalez14@ hotmail.com \\ Universidad Nacional de Pilar, Paraguay
}

\section{RESUMEN}

El trabajo tiene como objetivo determinar la Educación Financiera de Jóvenes Universitarios de la Facultad de Humanidades y Ciencias de la Educación, Periodo 20192020. El estudio se realiza en la Ciudad de Pilar, Neembucú. Se realizar una encuesta a los alumnos de la Universidad Nacional de Pilar, de tal manera a medir y evaluar la "Educación Financiera (Jóvenes Universitarios)" de la Facultad de Humanidades y Ciencias de la Educación (UNP), como resultado del propio proceso las dimensiones a analizar son: Medición y evaluación en Educación financiera. La presente investigación es abarcada desde una perspectiva fenomenológica, debido a que se enfoca a experiencias individuales subjetivas de los participantes, empleando un enfoque cuantitativo, el diseño de investigación implementado, es el preexperimental, transversal y descriptivo. los alumnos. Se ha llegado a la conclusión que rol del estado, de las instituciones educativa y también las familias, de formación y capacitación de manejo financiero, puesto que existe gran necesidad de información que la sociedad requiere temas específicos que puedan ayudar a tomar las mejores decisiones en la vida cotidiana y tener una vida más tranquila, considerando que se ha evidenciado la falta de una educación financiera en los jóvenes del grupo control.

Palabras clave: Educación Financiera; Jóvenes Universitarios; Facultad de Humanidades y Ciencias de la Educación. 


\title{
Financial Education of young university of the Faculty of Humanities and Educational Sciences, period 2019-2020
}

\begin{abstract}
The objective of the work is to determine the Financial Education of Young University Students of the Faculty of Humanities and Education Sciences, Period 2019-2020. The study is carried out in the City of Pilar, Neembucú. A survey will be carried out to the students of the National University of Pilar, in such a way as to measure and evaluate the "Financial Education (Young University Students)" of the Faculty of Humanities and Education Sciences (UNP), as a result of the process itself dimensions to be analyzed are: Measurement and evaluation in financial education. This research is covered from a phenomenological perspective, because it focuses on subjective individual experiences of the participants, using a quantitative approach, the implemented research design is the pre-experimental, transversal and descriptive. the students. It has been concluded that the role of the state, of educational institutions and also of families, of training and financial management training, since there is a great need for information that society requires specific topics that can help make the best decisions in daily life and have a calmer life, considering that the lack of financial education has been evidenced in the young people of the control group.
\end{abstract}

Keywords: Financial Education; University Students; Faculty of Humanities and Education Sciences.

Artículo recibido: 03 nov. 2020 Aceptado para publicación: 07 dic. 2020 Correspondencia ameliogonzalez14@ hotmail.com Conflictos de Interés: Ninguna que declarar 


\section{INTRODUCCIÓN}

La investigación estudia la Educación Financiera de Jóvenes Universitarios de la Facultad de Humanidades y Ciencias de la Educación, Periodo 2019-2020.

La Universidad Nacional de Pilar, surge en el año 1991, comenzando sus primeras actividades académicas gracias al apoyo del sector privado. Llega así el 14 de julio de 1991, en una Gran Asamblea Popular se resuelve fundar la "Universidad de Pilar en Formación”. La misma fue creada por Ley Número 529/94, aprobada por la Honorable Cámara de Senadores el 12 de Diciembre de 1994 y por la Honorable Cámara de Diputados el 15 de Diciembre de 1994, teniendo como sede la Ciudad de Pilar.

En la actualidad existen siete Facultades, las que se citan a continuación: Facultad de Ciencias Contables, Administrativas y Económicas, Facultad de Derecho, Ciencias Políticas y Sociales, Facultad de Ciencias Aplicadas, Facultad de Ciencias Agropecuarias y Desarrollo Rural, Facultad de Ciencias, Tecnologías y Artes, Facultad de Ciencias Biomédicas y la institución en estudio, la Faculta de Humanidades y Ciencias de la Educación, a fin de averiguar la cultura financiera y las diversas estrategias utilizadas por el mercado para ocasionar el endeudamiento masivo de los Jóvenes Universitarios de la Facultad de Humanidades y Ciencias de la Educación.

\section{EL PROBLEMA}

La educación financiera es un elemento capaz reducir la exclusión social y desarrollar el sistema financiero, además, se ha detectado que las personas desconocen los elementos y conceptos financieros básicos, lo que conlleva a tomar decisiones inadecuadas sobre ahorro, deuda e inversiones lo que puede perjudicar su bienestar personal, familiar actual y futuro.

Entre las causas que originan el problema es la falta de cultura financiera, es decir, proveer los gastos, elaborar un presupuesto acorde a la realidad de cada familia o persona como es el gasto para alimentación, vestimenta, educación, pequeños ahorros, la inversión, el crédito y los seguros, sino más bien están acostumbrados a gastar más de lo que recibe. Así también, debido al desarrollo acelerado de los mercados financieros, utilizan diversas promociones ya sean de tarjetas o endeudarse a través de proporciones ofrecidas por el mercado actual. 


\section{OBJETIVOS}

\section{General}

- Determinar la Educación Financiera de Jóvenes Universitarios de la Facultad de Humanidades y Ciencias de la Educación, Periodo 2019-2020.

\section{Específicos}

- Identificar la cultura financiera de Jóvenes Universitarios de la Facultad de Humanidades y Ciencias de la Educación.

- Describir las diversas estrategias utilizadas por el mercado para ocasionar el endeudamiento masivo de los Jóvenes Universitarios de la Facultad de Humanidades y Ciencias de la Educación.

\section{METODOLOGÍA.}

Entre algunas investigaciones similares se pueden citar una lista de Investigación, las que se citan a continuación.

\begin{tabular}{|c|c|c|c|c|}
\hline \multicolumn{5}{|c|}{ Investigación sobre temáticas similares } \\
\hline Autor (es) & Denominación & $\begin{array}{c}\text { Lugar de } \\
\text { Ana lisis }\end{array}$ & $\begin{array}{c}\text { Año de } \\
\text { Publicación }\end{array}$ & $\begin{array}{c}\text { Estrategias } \\
\text { Metodológicas }\end{array}$ \\
\hline $\begin{array}{l}\text { - Barbara Gisella } \\
\text { Cruz Vargas 1, } \\
\text { - Juan Carlos Díaz } \\
\text { Navarro 2, } \\
\text { - Mónica Noemí } \\
\text { Célleri Zúñiga } 3\end{array}$ & $\begin{array}{l}\text { Educación } \\
\text { Financiera }\end{array}$ & $\begin{array}{l}\text { Ecuador, } \\
\text { Guayaquil }\end{array}$ & 2016 & $\begin{array}{l}\text { Se realizaron las } \\
\text { búsquedas } \\
\text { siguientes: } \\
\text { a) aparición del } \\
\text { término "Educación } \\
\text { Financiera" } \\
\text { aparecidas } \\
\text { Google. } \\
\text { b) Revisión en } \\
\text { Scopus para el } \\
\text { término "finance } \\
\text { education", }\end{array}$ \\
\hline $\begin{array}{l}\text { - Edinson Cornejo } \\
\text { Saavedra } \\
\text { - Benito Umaña } \\
\text { Hermosilla } \\
\text { - Nataly Guiñez } \\
\text { Cabrera }\end{array}$ & $\begin{array}{l}\text { Endeudamiento } \\
\text { y educación } \\
\text { financiera del } \\
\text { adulto joven en } \\
\text { Chile }\end{array}$ & Chile, Chillán. & 2017 & $\begin{array}{l}\text { Endeudamiento y } \\
\text { educación financiera } \\
\text { del adulto joven en } \\
\text { Chile. }\end{array}$ \\
\hline
\end{tabular}




\begin{tabular}{|c|c|c|c|c|}
\hline $\begin{array}{l}\text { - Angela Araceli } \\
\text { Estrada } \\
\text { Martínez; } \\
\text { - Paula Arévalo } \\
\text { Guzmán; } \\
\text { - Shirley Jenine } \\
\text { Moya Pantoja; } \\
\text { - Claudia María } \\
\text { José Aranda } \\
\text { Magallanes; } \\
\text { - Stefanía Jeniffer } \\
\text { Rivera Sánchez. } \\
\text { Estudiantes de la } \\
\text { Universidad } \\
\text { Ricardo Palma }\end{array}$ & $\begin{array}{l}\text { Propuesta de } \\
\text { cuestionario } \\
\text { para medir el } \\
\text { nivel } \\
\text { de } \\
\text { conocimiento } \\
\text { sobre las } \\
\text { finanzas } \\
\text { personales } \\
\text { de jóvenes } \\
\text { universitarios. }\end{array}$ & $\begin{array}{c}\text { Perú, Santiago } \\
\text { de Surco }\end{array}$ & 2017 & $\begin{array}{l}\text { Se estructuró la base } \\
\text { teórica a partir del } \\
\text { análisis } \\
\text { de tesis, artículos y } \\
\text { libros. Además, se } \\
\text { realizó } \\
\text { una entrevista a un } \\
\text { profesional con } \\
\text { experiencia } \\
\text { laboral en el Sistema } \\
\text { financiero. }\end{array}$ \\
\hline $\begin{array}{l}\text { - PEÑARRETA } \\
\text { QUEZADA, } \\
\text { Miguel 1; } \\
\text { - GARCÍA } \\
\text { TINIZARAY, } \\
\text { Daisy 2; } \\
\text { - ARMAS } \\
\text { HERRERA, } \\
\text { Reinaldo } 3\end{array}$ & $\begin{array}{l}\text { Educación } \\
\text { financiera y } \\
\text { factores } \\
\text { determinantes: } \\
\text { Evidencias } \\
\text { desde Ecuador }\end{array}$ & Ecuador & 2019 & $\begin{array}{l}\text { Para lograr los } \\
\text { objetivos y dar } \\
\text { respuesta a las } \\
\text { hipótesis planteadas } \\
\text { se hace uso de dos } \\
\text { técnicas de análisis } \\
\text { multivariante: } \\
\text { análisis clúster y } \\
\text { análisis probit } \\
\text { multinomial. }\end{array}$ \\
\hline $\begin{array}{l}\text { - Milka Elena, } \\
\text { Escalera Chávez, } \\
\text { - Esmeralda } \\
\text { Tejada Peña, } \\
\text { - Arturo, García } \\
\text { Santillán }\end{array}$ & $\begin{array}{l}\text { Programas de } \\
\text { educación } \\
\text { financiera } \\
\text { implementados } \\
\text { en } \\
\text { américa latina }\end{array}$ & $\begin{array}{c}\text { Ecuador, } \\
\text { Guayaquil. }\end{array}$ & 2019 & \\
\hline $\begin{array}{c}\text { - Econ. Juan } \\
\text { López Vera. }\end{array}$ & $\begin{array}{l}\text { La (Des) } \\
\text { educación } \\
\text { Financiera en } \\
\text { Jóvenes } \\
\text { Universitarios } \\
\text { Ecuatorianos: } \\
\text { Una } \\
\text { Aproximación } \\
\text { Teórica. }\end{array}$ & $\begin{array}{c}\text { Ecuador, } \\
\text { Guayaquil. }\end{array}$ & 2016 & \\
\hline
\end{tabular}




\begin{tabular}{|c|c|c|c|c|}
\hline $\begin{array}{l}\text { - M. en A. Laura } \\
\text { Guillermina } \\
\text { Duarte Cáceres } \\
\text { - M. en F. Yolanda } \\
\text { Leonor Rosado } \\
\text { Muñoz } \\
\text { - M. en F. Martha } \\
\text { Isabel Bojórquez } \\
\text { Zapata }\end{array}$ & $\begin{array}{l}\text { La } \\
\text { competitividad } \\
\text { analítica de los } \\
\text { programas de } \\
\text { negocios de las } \\
\text { universidades }\end{array}$ & $\begin{array}{c}\text { México, Mérida, } \\
\text { Yucatán. }\end{array}$ & 2018 & $\begin{array}{l}\text { Se realizó un estudio } \\
\text { cuantitativo, } \\
\text { transversal, que a } \\
\text { través del } \\
\text { instrumento de la } \\
\text { encuesta se logra } \\
\text { conocer y analizar el } \\
\text { conocimiento, } \\
\text { actitud } \\
\text { comportamiento de } \\
\text { los estudiantes de } \\
\text { primer semestre } \\
\text { de la Maestría en } \\
\text { Finanzas de la } \\
\text { Universidad } \\
\text { Autónoma } \\
\text { Yucatán con respecto } \\
\text { a las Finanzas } \\
\text { Personales. }\end{array}$ \\
\hline \multicolumn{5}{|c|}{ Fuente: Elaboración propia en base a lecturas } \\
\hline
\end{tabular}

El estudio se realiza en la Ciudad de Pilar, Neembucú, una ciudad donde creció vertiginosamente, en cuanto a estudiantes en el departamento, existen 15 (quince) distrito, donde vienen a buscar su título terciario.

Se estudia a los jóvenes estudiantes de la Universidad Nacional de Pilar, de la Facultad de Humanidades y Ciencias de la Educación.

Se realizar una encuesta a los alumnos de la Universidad Nacional de Pilar, de tal manera a medir y evaluar la "Educación Financiera (Jóvenes Universitarios)" de la Facultad de Humanidades y Ciencias de la Educación (UNP), como resultado del propio proceso las dimensiones a analizar son: Medición y evaluación en Educación financiera. La presente investigación es abarcada desde una perspectiva fenomenológica, debido a que se enfoca a experiencias individuales subjetivas de los participantes, empleando un enfoque cuantitativo, el diseño de investigación a emplear, es el preexperimental, transversal y descriptivo. Preexperimental debido a que se limita a una descripción del fenómeno de la Educación Financiera, que experimentan los alumnos de la Universidad Nacional de Pilar, sin introducir ninguna modificación o alteración, según el tratamiento de la variable, 
es de corte transversal, ya que la información es recabada en un solo momento en el tiempo, utilizando formularios de encuesta, solicitar ,colaboración para el llenado de los mismos; es descriptivo ya que el propósito del presente trabajo es describir el grado de conocimiento de los alumnos universitarios, (de la Facultad de Humanidades y Ciencias de la Educación) sobre el tema abordado.

\section{REVISIÓN BIBLIOGRÁFICA (Marco Teórico)}

\section{Antecedentes de la Investigación}

La Educación Financiera se ha convertido en una necesidad de primera magnitud motivada en principio por las mismas condiciones actuales que llevan a que los ciudadanos tengan que manejar tanto a lo largo de su vida privada (Cooper \& Zhu, 2016). Según el estudio "Financial Literacy Among Youth in Latin America and the Caribbean" preparado por Mastercard en el año (2013, citado por Gómez 2016) se reveló que el 67\% de los Millennials en América Latina consideran que los conocimientos financieros que poseen son bajos. Esta cifra es alarmante dado que en la actualidad más de la mitad de la población de América Latina está por debajo de los 35 años de edad. Si a esto se suma que en dicho estudio se evidencia que el 74\% de los Millennials reconoce que la falta de entendimiento del Sistema Financiero es uno barrera importante para buscar o usar algún producto financiero que le permita cumplir sus metas.

Estudio del Instituto Nacional de la Juventud (INJUV, 2014) demostró que el 37\% de los jóvenes entre 18 y 24 años declaró tener deudas, préstamos o créditos a su nombre. Esta proporción aumentó a $47 \%$ en los jóvenes entre 25 y 29 años. El estudio también señaló que los principales ítems de endeudamiento fueron: crédito universitario (43\%); tarjetas de crédito (39\%); y créditos de consumo (30\%).

Zamalloa, (2017) en su estudio Educación y cultura financiera en emprendedores universitarios. Actualmente, entidades sin fines de lucro, privadas y del gobierno buscan proporcionar algún tipo de educación financiera a la población, no obstante, no tienen en cuenta como objetivo directo a estudiantes de nivel secundario (p. 8).

Tinoco (2018) considera que una buena práctica de los conocimientos en educación financiera llega a ser una herramienta fundamental para que el crédito en Perú no sea una necesidad permanente y que el endeudamiento no represente un estilo de vida; así como, enseñar que ahorrar e invertir, elaborar y administrar un presupuesto personal, genera 
riquezas para todo ciudadano en este país. En el Programa de Educación Financiera Global Microfinance Opportunities se distinguen tres conceptos importantes: Educación financiera: es aquella que transmite conocimiento, habilidades y actitudes necesarias con la finalidad de que las personas adopten buenas prácticas de manejo de dinero para la generación de ingreso, gasto, ahorro, endeudamiento e inversión (Gómez, 2009).

Alfabetización financiera: se define como la habilidad de tener la información necesaria para tomar decisiones efectivas sobre el uso y manejo del dinero (Gómez, 2009).

Capacidad financiera: es la que une a la educación y alfabetización financieras en un contexto de decisiones en el mundo real, constituyéndose en la habilidad que permite que la gente ante diversas opciones tome una decisión financiera informada (Gómez, 2009). Tendencia de la inclusión financiera

La tendencia de la inclusión financiera está enfocada en ofrecer a los niños y jóvenes el acceso y oportunidad de utilizar los productos y servicios financieros (CDS, 2013, citado por Escalera; Tejada \& García, 2017).

Los niños y jóvenes son considerados competentes financieramente cuando tienen el conocimiento para tomar decisiones financieras inteligentes. Child and Youth Finance International (CYFI) creó una teoría conocida como teoría del cambio (Billimoria, Penner y Knoote, 2013, Ibid), desarrollada por un grupo de académicos miembros de dicha organización. La teoría combina la educación financiera con la educación social, así como la inclusión financiera, con el fin de aumentar la capacidad financiera y la potenciación socioeconómica de los niños y jóvenes, para conducirlos a una mayor conciencia del entorno financiero (Escalera; Tejada \& García, 2017).

La capacidad de gestión de las finanzas personales se ha vuelto cada vez más importante para evaluar alternativas de ahorro y de crédito. No obstante, diferentes estudios han mostrado que los jóvenes no han recibido una buena educación en temas financieros (Cornejo; Umaña; Guiñez; Muñoz \& Mardones, 2017).

En el modelo, el grupo de trabajo propuso que la educación financiera, la educación social y la inclusión financiera son los componentes básicos de la potenciación y la capacidad financiera que sustentan en la responsabilidad económica para los niños y jóvenes, obteniendo como resultado reducir la pobreza, mejorar el bienestar económico y social sostenible, y proporcionar medios de subsistencia más sostenibles (Escalera; Tejada \& 
García, 2017). Aunque están relacionadas, estos términos se utilizan de maneras específicas y distintas en los programas e investigación.

La capacidad financiera es el conocimiento, habilidades financieras y acceso a los servicios financieros, que permiten a la gente a entender y actuar en sus mejores intereses económicos. La educación financiera incluye la instrucción y/o materiales diseñados para aumentar el conocimiento y las habilidades financieras. La educación social es la prestación de conocimientos y habilidades que cambian la comprensión y el conocimiento de los derechos de los individuos y de los derechos de los demás. Eso también implica el fomento de habilidades para la vida (Escalera; Tejada \& García, 2017, p 7).

La inclusión financiera es apropiada para el acceso, la calidad y accesibilidad a los servicios financieros. La potenciación es la sensación de confianza y eficacia que viven los niños y la juventud mediante el control de sus propias vidas, reclamando sus derechos. Con relación al constructo de inclusión financiera, se puede medir a través de varias dimensiones, incluida la accesibilidad, asequibilidad, conveniencia, flexibilidad, fiabilidad y seguridad (Escalera; Tejada \& García, 2017, p.8).

El conocimiento financiero de los jóvenes recibe una gran atención del área académica, siendo que existen evidencias sobre la escasa administración o manejo de principios básicos de las finanzas personales, tales como es la administración del gasto, ahorro, el uso del crédito, manejo de pago de interés, entre otros. Estos datos afirman las bajas tasas de ahorro, alto uso del crédito y sobrendeudamientos, son situaciones que indican que los adolescentes necesitan una preparación educativa que los ayude a tomar rumbo a este problema social por una parte siendo que afecta a muchas personas y personal al verse involucrado en situaciones de la que ha perdido todo control.

\section{Mercados financieros y endeudamientos}

La educación financiera ha venido tomando mayor relevancia en las últimas décadas.

Diferentes factores sociales, económicos y demográficos han impulsado esta tendencia. Dentro de estos se pueden mencionar:

- Endeudamiento creciente por parte de individuos debido a mayor oferta de créditos,

- Aumento en oferta y complejidad de elementos financieros

- Aumento de la inestabilidad laboral, 
- $\quad$ Aumento de la esperanza de vida, y

- $\quad$ Reducción de coberturas públicas de pensiones (Villada; López y Muñoz, 2017). En este contexto, una adecuada educación financiera es imperativa para mejorar el estándar de vida de los individuos. Según algunos expertos, fue precisamente la carencia de la misma la principal causa de la última crisis económica en Estados Unidos y Europa, originada en el mercado inmobiliario (Melvin y Taylor, 2009; Dwyer y Lothian, 2012; Bartram y Bodnar, 2009, Ibid). No se pueden desconocer los abusos del sistema financiero, pero fueron finalmente las personas naturales quienes tomaron la decisión de solicitar deudas por encima de sus posibilidades, adquirir una vivienda inflada de precio o invertir en títulos basura desconociendo la calidad de la inversión (Feldkircher, 2014, como se citó en Villada; López y Muñoz, 2017).

Quispe y Vilca (2019) consideran que la educación financiera es fundamental; pues nos familiariza con el uso del dinero y la gestión de nuestra economía personal. Los jóvenes y adolescentes tienen mayor capacidad de aprendizaje y retención por lo que emprender el tema de la educación financiera en su periodo formativo rendirá muchos frutos a lo largo de su vida.

Las prácticas constantes se convierten en habilidad y esto será posible en la medida de las prácticas en la administración de las finanzas personales, a través de presupuesto en el cual se estimen todos los ingresos y gastos para cada periodo establecido, pudiendo ser esta de manera mensual, trimestral, anual, etc, y cuando más temprano y en los diferentes estamentos se pueda aplicar estas prácticas, hablará mayor cantidad de personas con un manejo adecuado y prudente de sus finanzas.

\section{El endeudamiento financiero}

Identificar la trama de dispositivos vinculados con el endeudamiento financiero y comprender el modo en que opera sobre las subjetividades resulta particularmente relevante en vistas de la creciente financiarización (en su manifestación de uso masivo de tarjetas bancarias, no bancarias, tarjetas de compra, créditos y préstamos personales) que ha sostenido y canalizado el consumo popular en las últimas décadas (Hadad, 2019).

Existe un conjunto de características de la actividad bancaria que afectan la morosidad del sistema, entre ellas se puede mencionar, liquidez, rentabilidad, política crediticia, 
eficiencia en el manejo del riesgo (Ahumada y Budnevich, 2001; Salas y Saurina, 2003, como se citó en Hurtado y Altuve, 2018).

Además, de estos elementos de carácter microeconómico también existen factores macroeconómicos que explican el comportamiento y la tendencia de la morosidad del sistema, como ejemplo, cuando las economías entran en una fase recesiva, las empresas y las familias ven reducidos sus ingresos, también si la tasa de desempleo es elevada las familias ven disminuir sus posibilidades de obtener ingresos, lo que dificultaría poder cumplir con sus obligaciones crediticias, incluso con la existencia de políticas gubernamentales de subsidios y transferencias como las misiones sociales.

Según Estrada; Arévalo; Moya; Aranda y Rivera, (2017) gracias a una alfabetización financiera podríamos poner en práctica las finanzas personales adecuadas y convenientes según el interés propio, estos conocimientos financieros se deben fomentar desde las primeras etapas educativas de las personas.

Definen a las finanzas personales como toda decisión financiera y actividades que incluyen el presupuesto de ingresos y gastos de los hogares, ahorros, inversiones, hipotecas, seguros y todas las demás decisiones que requieren dinero, estableciendo objetivos a corto plazo y a largo plazo (Estrada; Arévalo; Moya; Aranda y Rivera, 2017). De acuerdo a estas revisiones bibliográficas se afirma que el consumismo y la falta de educación financiera ha ocasionado un desfasaje en el bienestar general de las personas y donde es necesario acatar en la juventud y por qué no decir en la niñez.

Publicación del Período Nacional Ultima Hora, octubre (2019) se ha presentado un proyecto de ley que establece la obligatoriedad de la educación financiera y finanzas personales en el currículo de la educación escolar básica y media en el sistema educativo nacional, este proyecto instala de manera obligatoria la disciplina de educación financiera dentro del sistema educativo. La malla curricular será confeccionada por el Ministerio de Educación y Ciencias (MEC), con la finalidad de otorgar al alumnado las herramientas y conocimientos necesarios para desarrollar un pensamiento crítico y objetivo a la hora de asumir compromisos financieros.

Una parte de la argumentación de dicho proyecto se basa en la necesidad de tener conocimientos sobre manejos financieros, siendo que las escuelas y universidades no la ofrecen normalmente este tipo de conocimiento; que el problema se agrava, ya que el 
entorno social presiona cada vez más para gastar, para consumir y no para ahorrar o invertir o alguna otra manera de generar bienestar económico de las personas. Datos que revelan la realidad de la problemática de las familias del país.

De acuerdo a un estudio de mayor Aylas, (2017) parte de la población no practica buenos hábitos financieros, y por ende las personas llegan a obtener malas decisiones con respecto al dinero.

Del Águila (2019) afirma que la ausencia de educación en finanzas provoca que las personas incurran en gastos excesivos, asuman riesgos innecesarios, y al no poner límites, pueden llegar a caer en endeudamientos, puesto que no poseen un presupuesto y caen fácilmente en créditos, comenzando por préstamos de parientes u otras personas, generando problemas financieros graves (p. 13).

Las finanzas personales se relacionan con el problema que enfrenta cada persona para operar sus recursos y tomar decisiones acerca de la forma más adecuada para manejar el patrimonio, satisfacer necesidades y tener claro que con cada decisión que se tome, se está administrando el proyecto de vida personal y familiar. El análisis de este tema parte de la teoría de los ciclos de la vida para el consumo y el ahorro, la cual establece que la familia percibe un flujo de ingresos durante su vida, que se extiende por varios períodos o años y por tanto, necesita seleccionar una trayectoria de consumo a través de su vida que sea consistente con sus ingresos a lo largo de este mismo lapso.

\section{ANÁLISIS Y DISCUSIÓN}

El endeudamiento financiero se presenta con más intensidad en la sociedad actual, debido a diversos factores como la falta de cultura financiera, lo que representa la inversión, previsión de gastos, elaboración de un presupuesto acorde a la realidad de cada familia y persona y la recesión económica; este presupuesto debe ser real y en la que se debe apuntar gasto para alimentación, educación, vestimenta, ahorros, la inversión, el crédito y los seguros, aun cuando el salario sea menor como culturas de otros países. 


\section{Cultura financiera - Estrategias utilizadas}

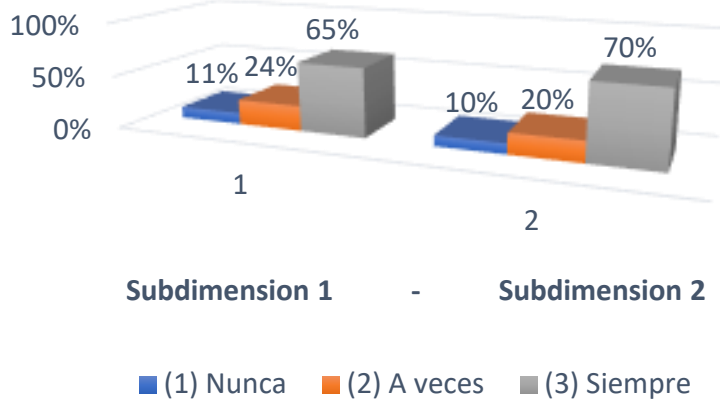

De igual manera la evaluación de los programas de educación financiera se convierte en herramienta fundamental, ya que permite medir la efectividad y el impacto, esto con el fin de hacer un mejor uso de los recursos existentes, revisar los contenidos y adaptar los canales utilizados para su entrega de acuerdo con los distintos públicos objetivo, (Banco Mundial, 2012).

En las investigaciones referentes a la educación financiera que se han realizado, presentan evidencia de que por lo general y no sólo los jóvenes, carecen de conocimientos en materia financiera, aunque tengan alguna formación académica, estos no manejan temas de elaboración de presupuestos, el efecto de la inflación y el dominio propio para no endeudarse a través de proporciones ofrecidas por el mercado actual.

Según CNMV (2008). En la actualidad, las personas se enfocan en formarse y formar a su familia en ganar dinero, pero no se preocupan por aprender cómo gastarlo y mucho menos en ahorrarlo como elemento de previsión y estabilización ante la incertidumbre del futuro. El sobreendeudamiento ha aumentado debido a la creciente tendencia de las personas de elevar su nivel de vida y satisfacer sus deseos adquiriendo productos y servicios que no tienen posibilidades económicas de solventar (Zakaria, Jaafar y Marican, 2012).

En este sentido, es esencial fomentar el ahorro como medio de prevención y convertirlo en un hábito que coadyuve al logro de objetivos tanto personales como familiares (Carrillo y Lamamié, 2008).

La educación financiera se vuelve fundamental en la elección de un producto financiero, para exigir derechos y cumplir obligaciones como clientes del Sistema Financiero, disminuyendo así la posibilidad de endeudamiento (Cohen y Nelson, 2011). 
La desinformación y falta de conocimiento sobre el tema financiero es una realidad que involucra a toda la sociedad, por lo que es necesario promover la educación financiera en los niños, jóvenes y adultos, (la familia) para que puedan tomar las mejores decisiones económicas, situación que se ha evidenciado mediante la encuesta aplicado a los jóvenes universitarios de la facultad en estudio.

En el Programa de Educación Financiera Global Microfinance Opportunities se distinguen tres conceptos importantes: Educación financiera: es aquella que transmite conocimiento, habilidades y actitudes necesarias con la finalidad de que las personas adopten buenas prácticas de manejo de dinero para la generación de ingreso, gasto, ahorro, endeudamiento e inversión (Gómez, 2009).

Alfabetización financiera: se define como la habilidad de tener la información necesaria para tomar decisiones efectivas sobre el uso y manejo del dinero (Gómez, 2009).

Capacidad financiera: es la que une a la educación y alfabetización financieras en un contexto de decisiones en el mundo real, constituyéndose en la habilidad que permite que la gente ante diversas opciones tome una decisión financiera informada (Gómez, 2009).

\section{CONSIDERACIÓN FINAL}

De acuerdo a Moreno, García y Gutiérrez, (2017) concluyen que el nivel de alfabetismo financiero entre los jóvenes de enseñanza media superior es bajo, como se ha registrado en otros países. En consecuencia, es importante considerar este resultado para el diseño de políticas públicas futuras que busquen modificar esta situación. Es necesario empezar a medir este nivel de alfabetismo financiero entre los jóvenes y en la población en general. El ahorro es uno de los aspectos más importantes en la vida económica de las personas, debido a que es la clave para obtener una independencia financiera y acumular riqueza. Además, el tener ahorros permite una seguridad económica y el poder cumplir metas como poner un negocio propio o adquirir algún bien como una vivienda o un automóvil. Los ahorros pueden tener diversos objetivos: ahorrar para cubrir costos de educación o servicios médicos, planeación para el retiro o poder afrontar emergencias de distinta índole (Zamora; García y Ramos, 2018).

Crear o incursionar en la malla curricular de la Educación Escolar Básica es una buena posibilidad de educar a los niños desde temprana edad, considerando que es un tema que debe de ser abordada desde a nivel nacional y llegar en los hogares y empezar a educar a 
la población más joven, considerando que han llegado a la vida universitario y conforme a los hallazgos, es esta investigación muchos de los jóvenes universitario no poseen una cultura financiera.

Aunado a esta propuesta de mejora curricular en temas de educación financiera, se resalta la necesidad de adoptar una metodología que permita evaluar la eficiencia y eficacia de los programas que se implementen en las escuelas, en general, y en particular de un programa que contenga temas de educación financiera (Moreno, García y Gutiérrez, 2017).

Existen planes curriculares implementadas en otros países cuyo principales ejes temáticos es la educación financiera, consumo responsable, comercio justo, mercadeo, servicio al cliente y contabilidad con ello se busca el desarrollo de las competencias relacionadas con estos temas que impide que las personas participen activa y responsablemente en los procesos económicos ya sea en el corto, mediano y largo plazo, influyendo de esta manera negativamente sobre el bienestar individual y familiar, retrasando con ello el avance de la sociedad en su conjunto, (Silva, et al., 2017).

Los resultados obtenidos en investigaciones permiten concluir que los estudiantes universitarios no saben calcular tasas de interés, de ahí que al interpretar la información relacionada con el precio de los bonos en donde se involucran cálculos asociados a tasas de interés, demuestran un bajo nivel de educación financiera. El alumno no la considera en el cálculo de valuación del dinero, por lo que no protege sus ahorros del efecto que ésta pueda tener en su patrimonio (Moreno, García y Gutiérrez, 2017).

En dicho reporte se concluye que las personas se enfocan en enseñar a ganar dinero, pero no enseñan cómo gastarlo, y mucho menos cómo ahorrarlo, como medio de previsión y estabilidad, especialmente en tiempos de crisis, cuando es primordial fomentar el ahorro y convertirlo en un hábito y un medio para el logro de objetivos personales y familiares (Moreno, García y Gutiérrez, 2017).

Otros resultados con los que se han hallado por otros estudios en diferentes partes del mundo, ponen de manifiesto la necesidad de una mayor penetración curricular de la educación financiera. Incluir en los programas de educación media del país temas que contribuyan a mejorar en las personas el manejo del dinero en el tiempo, que los preparen para optimizar su presupuesto y los acerquen a la adecuada identificación de fuentes de 
financiamiento, entre otras, conllevará a mejorar la dinámica económica familiar y a mejorar las prácticas de consumo y ahorro en pro del crecimiento económico (Moreno, García y Gutiérrez, 2017).

De manera general, se concluye el rol del estado, de las instituciones educativa y también las familias, de formación y capacitación de manejo financiero, puesto que existe gran necesidad de información que la sociedad requiere temas específicos que puedan ayudar a tomar las mejores decisiones en la vida cotidiana y tener una vida más tranquila, considerando que se ha evidenciado la falta de una educación financiera en los jóvenes del grupo control.

\section{REFERENCIAS BIBLIOGRAFICAS}

Arrubla, M. (2016). Finanzas y educación financiera en las empresas familiares Pymes. Sinapsis (8), 99- 118.

Aylas, Y. (2017). Análisis de la relación entre la educación financiera y variables de la tarjeta de crédito en los estudiantes de la modalidad presencial de la Facultad de ciencias de la empresa de la Universidad Continental - Huancayo, 2017.

Banco Mundial. (2012). Evaluaciones de políticas nacionales de Educación: La Educación superior en Colombia. /Banco\%20 Mundial_2012.

Carrillo, C. y J. Lamamié (2008), Educación financiera y ahorro familiar. Implicaciones de la crisis financiera global.

Cohen, M. y C. Nelson (2011), Financial literacy: a step for clients towards financial inclusion, en Global Microcredit Summit, Commissioned Workshop Paper, noviembre. Comisión Nacional del Mercado de Valores (cnmv) (2008), "Plan de Educación Financiera 2008-2012”, Madrid, Banco de España.

Cooper, R., et al.Zhu, G. (2016). Household finance over the life-cycle: What does education contribute? Review of Economic Dynamics, 20, 63-89. doi: 10.1016/j.red.2015.12.001

Cornejo-Saavedra, E; Umaña-Hermosilla, B; Guiñez-Cabrera, N; Muñoz-Silva, D y Mardones-Lagos, C. (2017). Endeudamiento y educación financiera del adulto joven en Chile.Young adult debt rate and financial education in Chile. Universidad del Bio Bío, Facultad de Ciencias. Empresariales, Departamento de Gestión. Empresarial, Avenida Andrés Bello N720, Chillán, CHILE. 
Del Aguila, M. (2019). La Educación financiera de la población económicamente activa de la asociación urbanización. Buenos Aires afilador Tingo María, periodo 2018.

Escalera-Chávez, M; Tejada-Peña E y García-Santillán; A. (2017). Uso de los servicios financieros. Estudio empírico en estudiantes universitarios. International Journal of Developmental and Educational Psychology INFAD Revista de Psicología de Hoy: Retos, Logros y Perspectivas de Futuro. Psicología de la Adolescencia, México.

Estrada Martínez, A; Arévalo Guzmán, P; Moya Pantoja, S; Aranda Magallanes, C.M y Rivera Sánchez, S. (2017). Propuesta de Cuestionario para Medir el Nivel de Conocimiento sobre las Finanzas Personales de Jóvenes Universitarios. Proposal Of A Questionnaire To Measure The Knowledge Level Of Personal Finances In University Students. Estudiantes de la Universidad Ricardo Palma. Facultad de Ciencias Económicas y Empresariales. Escuela Profesional de Administración de Negocios Globales

Franz M. Gómez-Soto (2009). Educación Financiera: Retos y Lecciones a partir de Experiencias Representativas en el Mundo Documento Preparado para el Proyecto Capital Bogotá, Colombia.

Hadad, Iara. (2019). La construcción social y técnica de la deuda morosa. Revista mexicana de sociología, 81(1), 89-115. Recuperado de http://www.scielo.org.mx/scielo.php?script=sci_arttext\&pid=S018825032019000100089\&lng=pt\&tlng=pt

Hurtado Briceño, A y Altuve, J. (2018). Análisis de los factores que influyen en la morosidad del sistema bancario venezolano (2005-2015). Revista Venezolana de Análisis de Coyuntura, vol. XXIV.

Instituto Nacional de la Juventud - INJUV (2014). Opinión Pública Joven. Serie de Estudios INJUV, Vol. 8, 170 págs. Recuperado de http://www.injuv. gob.cl/portal/wp-content/files_mf/libroestudios.

Moreno García, E; García Santillán M y Gutiérrez Delgado, L (2017). Nivel de educación financiera en escenarios de educación superior. Un estudio empírico con estudiantes del área económico-administrativa -México. 
Periódico Ultima Hora (2019). Obligatoriedad de educación financiera en escuelas y colegios. Recuperado de: https://www.google.com/search?q=Per\%C3\%ADodo+Nacional+Ultima+Hora, +octubre+(2019), +educacion+financiera\&spell=1\&sa=X\&ved=2ahUKEwiyhu F-pnoAhVRA9QKHQSyCrUQBSgAegQICBAn - 2 de octubre de 2019

Quispe, Y., \& Vilca, M. (2019). Influencia de una educación financiera de la comunidad escolar en la calidad de vida de los estudiantes y padres de la institución educativa particular. Tomas Marsano nivel secundario distrito de Alto Selva Alegre Arequipa - 2018.

Silva Giraldo, C. A., Celis Salazar, O., \& Rangel, A. N. (2017). Diseño de un Sistema de Medición de Impacto Social del Proyecto de Educación Financiera desarrollado en Uniminuto, Bucaramanga durante el año 2.016. Memorias VI Encuentro Internacional de Investigación y Espíritu Empresarial.

Tinoco, W. S. (2018). Educación financiera en estudiantes universitarios de una universidad del departamento de Junín - 2017. Universidad César Vallejo. Retrieved from http://repositorio.ucv.edu.pe/handle/UCV/16117

Villada, F; López-Lezama, J; Muñoz-Galeano, N. (2017). El Papel de la Educación Financiera en la Formación de Profesionales de la Ingeniería Formación Universitaria, vol. 10, núm. 2, 2017, pp. 13-22. Centro de Información Tecnológica La Serena, Chile.

Villagómez, F, (2016). Alfabetismo financiero en jóvenes preparatorianos en la Zona Metropolitana del Valle de México. El Trimestre Económico, vol. LXXXIII(3), núm. 331, julio-septiembre, 2016, pp. 677-706 Fondo de Cultura Económica Distrito Federal, México

Zakaria, R., N. Jaafar y S. Marican (2012), Financial behavior and financial position: a structural equation modelling approach, en Middle-East Journal of Scientific

Zamalloa, J. (2017). Inclusión financiera en Perú: Educación y cultura financiera en $\begin{array}{lll}\text { emprendedores } & \text { universitarios. }\end{array}$ http://dialnet.unirioja.es/servlet/articulo?codigo=257082\&info=resumen\&idio $\underline{\mathrm{ma}=\mathrm{SPA}}$ 
Zamora Lobato, T; García Santillán, A; Ramos Hernández, J. (2018). Algunas Características que Explican. El Comportamiento de los Jóvenes Universitarios Hacia El Ahorro International Journal of Developmental and Educational Psychology, núm. 2, 2018 Asociación Nacional de Psicología Evolutiva y Educativa de la Infancia, Adolescencia y Mayores, España. 